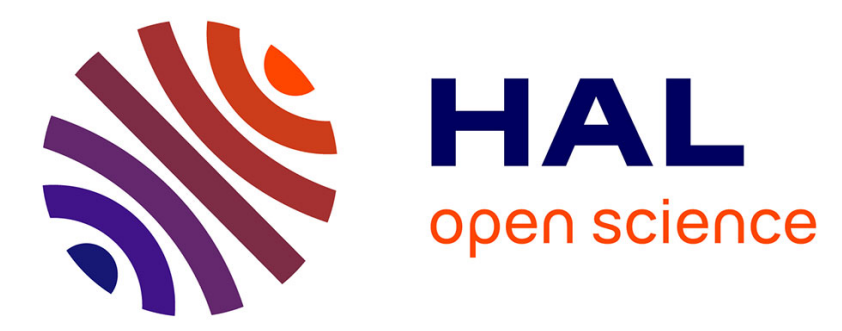

\title{
Optimal Area Power Efficiency in Cellular Networks
}

Bhanukiran Perabathini, Marios Kountouris, Mérouane Debbah, Alberto

Conte

\section{To cite this version:}

Bhanukiran Perabathini, Marios Kountouris, Mérouane Debbah, Alberto Conte. Optimal Area Power Efficiency in Cellular Networks. 2014 IEEE Globecom Workshops (GC Wkshps), Dec 2014, Austin, United States. 10.1109/glocomw.2014.7063589 . hal-01098896

\section{HAL Id: hal-01098896 https://hal.science/hal-01098896}

Submitted on 29 Dec 2014

HAL is a multi-disciplinary open access archive for the deposit and dissemination of scientific research documents, whether they are published or not. The documents may come from teaching and research institutions in France or abroad, or from public or private research centers.
L'archive ouverte pluridisciplinaire HAL, est destinée au dépôt et à la diffusion de documents scientifiques de niveau recherche, publiés ou non, émanant des établissements d'enseignement et de recherche français ou étrangers, des laboratoires publics ou privés. 


\title{
Optimal Area Power Efficiency in Cellular Networks
}

\author{
Bhanukiran Perabathini ${ }^{1}$, Marios Kountouris ${ }^{2}$, Mérouane Debbah ${ }^{2}$ and Alberto Conte ${ }^{1}$ \\ ${ }^{1}$ Alcatel-Lucent Bell Labs, 91620 Nozay, France \\ ${ }^{2}$ Department of Telecommunications, SUPELEC, 91192 Gif-sur-Yvette, France \\ ${ }^{3}$ Alcatel-Lucent Chair, SUPELEC, 91192 Gif-sur-Yvette, France \\ \{bhanukiran.perabathini, alberto.conte\}@alcatel-lucent.com, \\ \{marios.kountouris, merouane.debbah\}@ supelec.fr
}

\begin{abstract}
In this paper, we study the problem of minimizing the area power consumption in wireless cellular networks. We focus on the downlink of a single-tier network, in which the locations of base stations (BSs) are distributed according to a homogeneous Poisson point process (PPP). Assuming that a mobile user is connected to its strongest candidate BS, we derive bounds on the optimal transmit power in order to guarantee a certain minimum coverage and data rate. Under the same quality of service constraints, we find the optimal network density that minimizes the area power density. Our results show that the existence of an optimal BS density for minimizing the power consumption depends on the value of the pathloss exponent.

Index Terms-Cellular networks, green wireless communication, Poisson point process, area power density, energy efficiency, optimal base station density.
\end{abstract}

\section{INTRODUCTION}

In wireless cellular networks, the galloping demand for connectivity, data rate, and quality of service (QoS) [1] cannot be satisfied merely by increasing indefinitely the transmit power of the base stations (BSs). This is mainly due to the fact that an increase in transmit power, besides increasing the signal strength from the desired BS, also increases the interference received by the non-serving BSs. This may effectively decrease the signal-to-interference-plus-noise ratio (SINR) experienced by the user terminal, thus having a negative impact on the QoS. Besides that, it is also essential from an energy efficiency perspective to address the problem of minimizing the energy expenditure while maintaining certain constraints such as target coverage and minimum data rate.

There are various approaches in order to address this problem. For instance, if a finite number of BSs are deployed in a regular hexagonal (or grid) cellular fashion [2], one might seek to minimize the total power consumed by obtaining the optimal operating parameters, such as the hexagonal cell size and the magnitude of transmit power at each BS, while guaranteeing a certain QoS. Nevertheless, this approach involves very cumbersome analysis as evaluating the spatial distribution of the SINR in a grid-based model becomes prohibitively complex as the system size increases and one may have to resort to extensive simulations. A common simplification in modeling cellular networks which enables us handle the problem analytically is to assume that the locations of BSs are randomly scattered on a two dimensional plane surface according to a homogeneous Poisson point process (PPP) [3]. Several works studied the validity of PPP modeling of BSs (in comparison with regular cellular models,) and it is often shown to provide useful insights into the statistical behavior of key performance metrics [4], [5].

In this paper, we consider a single-tier cellular network, in which the BS locations are modeled according to a homogeneous spatial PPP. We assume that a mobile user is connected to the BS that provides the highest SINR and we impose two QoS constraints, namely a target coverage probability and a target minimum average rate experienced by the typical user. We aim at deriving the optimal BS density that maximizes the power efficiency, i.e. minimizes the power consumption per unit area. Evidently, a network is power efficient if the area power consumption decreases with increasing the BS density or reducing the cell size. Most prior work analyzed the performance of single-tier or heterogeneous Poisson cellular networks in terms of energy efficiency [6], [7], [8]. The most related work is [9], in which the authors analyze the impact of transmit power reduction on the area power consumption of the network under closest $\mathrm{BS}$ association. In this work, under strongest BS association, we derive bounds on the optimal transmit power in order to guarantee a certain minimum coverage and data rate. Under the same quality of service constraints, we find the optimal network density that minimizes the area power consumption, whose existence depends on the pathloss exponent and the target QoS guarantees.

The remainder of the paper is organized as follows: In Section II, we present the system model and motivate the optimization problem. In Section III, we provide bounds on the optimal transmit power to minimize the area power consumption under minimum coverage and rate constraints. In Section IV, we evaluate the optimal BS density that minimizes the area power consumption and simulation results are given in Section V. Section VI concludes the paper.

\section{System ModeL}

\section{A. Network model}

We consider the downlink of a single-tier cellular network, in which the locations of BSs are distributed on a twodimensional Euclidean plane $\mathbb{R}^{2}$ according to a homogeneous 
PPP $\Phi=\left\{r_{i}\right\}_{i \in \mathbb{N}}$ with density $\lambda_{b}$, where we denote by $r_{i} \in \mathbb{R}^{2}$ the location of the $i$-th BS. We assume that the users are also randomly distributed according to an independent PPP of density $\lambda_{u}$, such that $\lambda_{u} \geq \lambda_{b}$. Without loss of generality, we focus on a mobile (typical) user at the origin for calculating the performance metrics of interest, i.e. coverage probability and rate. The total bandwidth is denoted by $B$ and the bandwidth per user is given by $B_{u}=B \frac{\lambda_{b}}{\lambda_{u}}$. We assume that all BSs transmit with the same constant power $P$ and an additional operational power $P_{o}$ (e.g. due to hardware and signaling) is consumed at each BS. In such a system, the power expenditure per unit area, coined as area power consumption (APC), is given as

$$
\mathcal{P}=\lambda_{b}\left(P+P_{o}\right) .
$$

We model the system under consideration such that the transmitted signal from a given BS is subject to two propagation phenomena before it reaches the user: (i) a distance-dependent pathloss governed by the pathloss attenuation function $g(r)=$ $b r^{-\alpha}$, where $b$ is the pathloss coefficient and $\alpha$ is the pathloss exponent (ii) Rayleigh fading with mean 1 . According to the above assumptions and notation, the signal strength from $i$-th $\mathrm{BS}$ as received by the reference user is given as

$$
p_{i}\left(r_{i}\right)=h_{i} P b r_{i}^{-\alpha} .
$$

We further assume the presence of noise in the medium with power variance

$$
\sigma^{2}=\beta \lambda_{b},
$$

with $\beta=B \frac{1}{\lambda_{u}} \frac{F k T}{b}$, where $F$ is the receiver noise figure, $k$ is Boltzmann constant, and $T$ is the ambient temperature. If the reference user is connected to the $i$-th $\mathrm{BS}$, it receives a signal of power $p_{i}\left(r_{i}\right)$ from it. The sum of the received powers from the remaining BSs contributes to interference to this signal. As a result, the received SINR at the reference user when served by the $i$-th $\mathrm{BS}$ is given by

$$
\mathrm{SINR}=\frac{h_{i} g\left(r_{i}\right) P}{\sigma^{2}+I_{i}},
$$

where, $I_{i}=\sum_{r_{j} \in \Phi \backslash r_{i}} p_{j}\left(r_{j}\right)$.

In a downlink scenario, although the reference user can technically be served from any BS, a connection with a particular BS has to be established according to an association policy to ensure QoS, which may have impact on the APC optimization. In this work, we assume that a mobile user connects to the strongest BS, i.e. the BS that provides the maximum received SINR. The reference user is said to be covered when there is at least one BS that offers an SINR $>\gamma$. If no BS offers an SINR greater than this threshold, we say that the reference user is on coverage outage. We assume the condition $\gamma>1$, which is needed to ensure that there is only one BS that serves the required level of SINR at a given instant [10].

\section{B. Problem formulation}

The objective of this paper is to obtain the optimal BS density $\lambda_{b}$ and transmit power $P$ so that the area power consumption $\lambda_{b}\left(P+P_{0}\right)$ is minimized subject to a minimum coverage probability constraint and a minimum data rate guarantee. As stated above, the coverage probability, $P_{\mathrm{cov}}$, is defined as the probability that the reference user is covered. Following the aforementioned definition, the coverage probability is given as the probability that the SINR received by the reference user on an average is greater than $\gamma$. The user data rate per unit bandwidth, $R$ is defined as the expectation value of $B \frac{\lambda_{b}}{\lambda_{u}} \log _{2}(1+\mathrm{SINR})$.

\section{OPTIMAL POWER FOR TARGET COVERAGE AND RATE}

In this section we address the following optimization problem:

$$
\begin{array}{ll}
\underset{P \in(0, \infty)}{\arg \min } & \mathcal{P}=\lambda_{b}\left(P+P_{o}\right) \\
\text { s.t. } & \begin{cases}(i) & P_{\mathrm{cov}} \geq \epsilon P_{\mathrm{cov}}^{\mathrm{NN}} \\
(i i) & R \geq \delta R^{\mathrm{NN}}+R_{\text {min }}\end{cases}
\end{array}
$$

where $P_{\mathrm{cov}}^{\mathrm{NN}}$ and $R^{\mathrm{NN}}$ are the coverage probability and the peruser rate, respectively at the no noise regime, and $\epsilon, \delta$ are positive numbers. Note that when the transmit power is infinity (no noise case), the coverage probability is scale invariant, i.e. the coverage probability and also the spectral efficiency do not depend on the BS density.

Lemma 1. If $P_{c}^{*}$ is the minimum transmit power that satisfies the constraint $P_{\text {cov }} \geq \epsilon P_{c o v}^{N N}$, then $P_{c}^{*} \geq \frac{A_{1}}{\lambda_{b}^{\frac{\alpha}{2}-1}}$, where $A_{1}=$ $\frac{\beta \Gamma\left(1+\frac{\alpha}{2}\right)}{b C^{\frac{\alpha}{2}}(\alpha)(1-\epsilon)}$ and $C(\alpha)=\frac{2 \pi^{2}}{\alpha} \operatorname{cosec} \frac{2 \pi}{\alpha}$.

Proof. The coverage probability under strongest BS association for a general pathloss function $g(r)$ is given as [10], [11]

$$
\begin{aligned}
P_{\text {cov }}\left(P, \lambda_{b}\right) & =\mathbb{P}[\operatorname{SINR} \geq \gamma] \\
& =\pi \lambda_{b} \int_{0}^{\infty} \exp \left(-q\left(\gamma, \lambda_{b}, r\right)\right) d r
\end{aligned}
$$

where

$$
q\left(P, \lambda_{b}, r\right)=\frac{\gamma \sigma^{2}}{P g(\sqrt{r})}+\lambda_{b} \int_{0}^{\infty} \frac{\pi \gamma g\left(\sqrt{r_{i}}\right)}{g(\sqrt{r})+\gamma g\left(\sqrt{r_{i}}\right)} d r_{i} .
$$

We use the standard pathloss model $g(r)=b r^{-\alpha}$ and incorporate it in (6) and (5) to get the expression for coverage probability as

$$
P_{\operatorname{cov}}\left(P, \lambda_{b}\right)=\pi \lambda_{b} \int_{0}^{\infty} \exp \left[-\lambda_{b} \frac{\gamma \beta r^{\frac{\alpha}{2}}}{P b}-\lambda_{b} C(\alpha) \gamma^{\frac{2}{\alpha}} r\right] d r
$$

where $C(\alpha):=\frac{2 \pi^{2}}{\alpha} \operatorname{cosec} \frac{2 \pi}{\alpha}$. In the case of low noise $\left(\sigma^{2} \rightarrow 0\right)$, the above expression can be simplified by using the approximation $e^{-x} \approx 1-x$.

$$
\begin{aligned}
P_{\operatorname{cov}}\left(P, \lambda_{b}\right) & \approx \pi \lambda_{b} \int_{0}^{\infty}\left(1-\frac{\gamma \beta \lambda_{b} r^{\frac{\alpha}{2}}}{P b}\right) e^{-\lambda_{b} C(\alpha) \gamma^{\frac{2}{\alpha}} r} d r \\
& =P_{\operatorname{cov}}^{\mathrm{NN}}\left(1-\frac{\beta \Gamma\left(1+\frac{\alpha}{2}\right)}{P b \lambda_{b}^{\frac{\alpha}{2}-1} C^{\frac{\alpha}{2}}(\alpha)}\right)
\end{aligned}
$$


where $P_{\mathrm{cov}}^{\mathrm{NN}}:=\frac{\pi}{\gamma^{\frac{2}{\alpha}} C(\alpha)}$ is the coverage probability observed by the reference user in the case of negligible noise. Fig. 1 gives a justification to the above approximation by comparing the numerical plots of coverage probability before and after the approximation. By substituting the expression for $P_{\mathrm{cov}}$ from (8) into the constraint equation $P_{\text {cov }} \geq \epsilon P_{\text {cov }}^{\mathrm{NN}}$ we get a condition on the range of optimal transmit power $P_{c}^{*}$ as

$$
P_{c}^{*} \geq \frac{A_{1}}{\lambda_{b}^{\frac{\alpha}{2}-1}}
$$

where $A_{1}=\frac{\beta \Gamma\left(1+\frac{\alpha}{2}\right)}{b C^{\frac{\alpha}{2}}(\alpha)(1-\epsilon)}$. This equation establishes the approximate minimum transmit power as a function of $\lambda_{b}$ that satisfies the coverage constraint.

Lemma 2. If $P_{r}^{*}$ is the minimum transmit power to satisfy $R \geq R_{\text {min }}+\delta R^{N N}$, then $P_{r}^{*} \geq \frac{A_{2}(\lambda)}{\lambda_{b}^{\frac{\alpha}{2}}}$, where $A_{2}\left(\lambda_{b}\right)=$ $\frac{\sigma^{2} \Gamma\left(1+\frac{\alpha}{2}\right)}{b C^{\frac{\alpha}{2}}(\alpha)\left(1-\delta B \frac{\lambda_{b}}{\lambda u}\right)}$.

Proof. The per-user rate is analytically given as

$$
\begin{aligned}
R & =B \frac{\lambda_{b}}{\lambda_{u}} \mathbb{E}\left[\log _{2}(1+\mathrm{SINR})\right] \\
& =R_{\min }+B \frac{\lambda_{b}}{\lambda_{u}} \int_{\ln 2}^{\infty} \mathbb{P}\left[\mathrm{SINR} \geq e^{t}-1\right] d t
\end{aligned}
$$

where $R_{\min }$ is the minimum rate [10].

As in eq (10), the rate per BS per unit bandwidth experienced by the reference user is given by

$$
\begin{aligned}
R & =R_{\min }+B \frac{\lambda_{b}}{\lambda_{u}} \mathbb{E}[\log (1+\mathrm{SINR})] \\
& =R_{\min }+B \frac{\lambda_{b}}{\lambda_{u}} \int_{t>\ln 2} \mathbb{P}\left[\operatorname{SINR}>e^{t}-1\right] d t \\
& =R_{\min }+B \frac{\lambda_{b}}{\lambda_{u}} \pi \lambda_{b} \int_{0}^{\infty} \int_{\ln 2}^{\infty} \exp \left[-\frac{\sigma^{2} r^{\frac{\alpha}{2}}}{P b}\left(e^{t}-1\right)\right. \\
& \approx R_{\min }+B \frac{\lambda_{b}}{\lambda_{u}}\left(\frac{\pi}{C(\alpha)} \int_{\ln 2}^{\infty}\left(e^{t}-1\right)^{-\frac{2}{\alpha}} d t\right. \\
& \left.-\frac{\pi \sigma^{2} \lambda_{b}}{P b} \int_{\ln 2}^{\infty} \frac{\left(e^{t}-1\right)^{-\frac{2}{\alpha}} \Gamma\left(1+\frac{\alpha}{2}\right)}{C^{1+\frac{\alpha}{2}}(\alpha) \lambda_{b}^{\frac{\alpha}{2}}} d t\right) \\
& =R_{\min }+B \frac{\lambda_{b}}{\lambda_{u}} R^{\mathrm{NN}}\left(1-\frac{\beta \Gamma\left(1+\frac{\alpha}{2}\right)}{P b \lambda_{b}^{\frac{\alpha}{2}-1} C^{\frac{\alpha}{2}}(\alpha)}\right)
\end{aligned}
$$

where $R^{\mathrm{NN}}:=\frac{\alpha \pi 2^{\frac{\alpha-2}{2}}{ }_{2} F^{1}\left(\frac{2}{\alpha}, \frac{2}{\alpha}, \frac{2+\alpha}{\alpha}, \frac{1}{2}\right)}{C(\alpha)}$, is the rate per user when the noise is negligible. By substituting the expression for rate $R$ in the constraint equation $R \geq \delta R^{\mathrm{NN}}+R_{\min }$ to get a condition on the optimal transmit power $P_{r}^{*}$ as

$$
P_{r}^{*} \geq \frac{A_{2}\left(\lambda_{b}\right)}{\lambda_{b}^{\frac{\alpha}{2}-1}}
$$

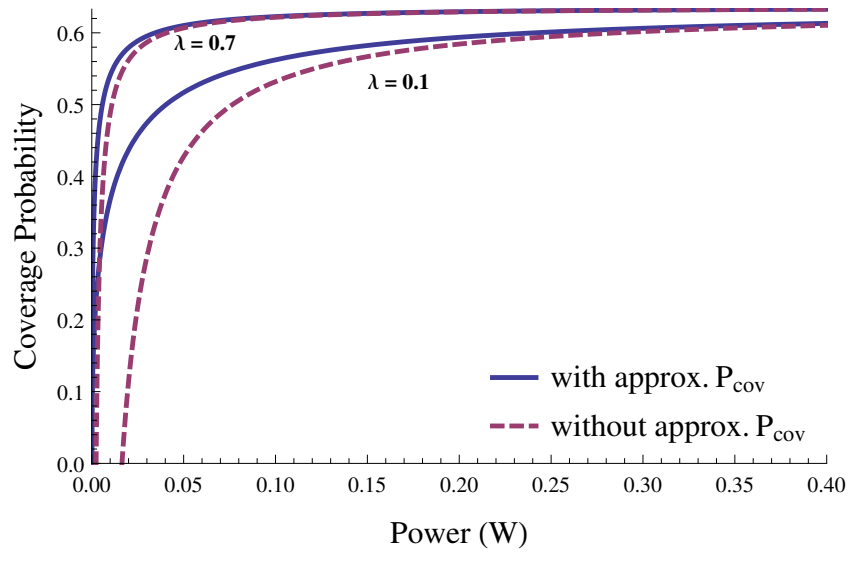

Figure 1. Coverage probability vs. Transmit Power $P$ with and without approximation made in (8); for two different values of $\lambda_{b}, \beta=10^{-3}, \lambda_{u}=$ $0.01 \mathrm{~m}^{-2}, B=20 \times 10^{6} \mathrm{~Hz}, \alpha=4$. The curves almost coincide for lower values of $\beta$.

where $A_{2}\left(\lambda_{b}\right)=\frac{\beta \Gamma\left(1+\frac{\alpha}{2}\right)}{b C^{\frac{\alpha}{2}}(\alpha)\left(1-\delta \frac{\lambda u}{B \lambda_{b}}\right)}$. This equation establishes the approximate minimum transmit power as a function of $\lambda_{b}$ that satisfies the rate constraint.

It follows naturally that the optimal transmit power that satisfies both the conditions (9) and (12) will therefore be $P^{*}=\max \left\{P_{c}^{*}, P_{r}^{*}\right\}$.

\section{OPTIMAL BS DENSITY}

Since the objective of our optimization is to minimize the APC $(\mathcal{P})$, we seek to minimize the function

$$
\begin{aligned}
\mathcal{P}\left(\lambda_{b}\right) & =\left(P^{*}+P_{o}\right) \lambda_{b} \\
& =\frac{\max \left\{A_{1}, A_{2}\left(\lambda_{b}\right)\right\}}{\lambda_{b}^{\frac{\alpha}{2}-1}}+P_{o} \lambda_{b}
\end{aligned}
$$

with respect to $\lambda_{b}$.

Following eq (13), there are two possible expressions for $\mathcal{P}$ depending on which is larger between $A_{1}$ and $A_{2}\left(\lambda_{b}\right)$, which in turn depends on the value of $\lambda_{b}$.

We study the two cases $A_{1}>A_{2}\left(\lambda_{b}\right)$ and $A_{1}<A_{2}\left(\lambda_{b}\right)$ and proceed with the optimization of $\mathcal{P}$ with respect to $\lambda_{b}$ in each case.

Case 1: If $A_{1}>A_{2}\left(\lambda_{b}\right)$ then $\lambda_{b} \in\left[\frac{\delta \lambda_{u}}{\epsilon B}, \infty\right)$ and the optimal transmit power $P^{*}=P_{c}^{*}$.

Therefore, the APC at optimal power follows as

$$
\mathcal{P}\left(\lambda_{b}\right)=\frac{\beta \Gamma\left(1+\frac{\alpha}{2}\right)}{b C^{\frac{\alpha}{2}}(\alpha)(1-\epsilon)} \frac{1}{\lambda_{b}^{\frac{\alpha}{2}-2}}+P_{o} \lambda_{b} .
$$

This is clearly a convex function in $\lambda_{b}$ for $\alpha>2$. Therefore, we differentiate $\mathcal{P}\left(\lambda_{b}\right)$ with respect to $\lambda_{b}$ and solve it for the optimum $\lambda_{b}^{*}$

$$
\frac{d \mathcal{P}\left(\lambda_{b}\right)}{d \lambda_{b}}=0 \Rightarrow \lambda_{b}^{*}=\left[\frac{\beta \Gamma\left(1+\frac{\alpha}{2}\right)}{b C^{\alpha}(\alpha)(1-\epsilon)} \frac{\left(\frac{\alpha}{2}-2\right)}{P_{o}}\right]^{\frac{\alpha}{2}-1}
$$

It can be noticed that $\lambda_{b}^{*}=0$ for $\alpha=4$ which means that when $\alpha=4$ the optimal BS density has only the 
trivial solution. We comment further on the relation between the existence of optimum and the pathloss exponent $\alpha$ in Section V, where we analyze (15) numerically and compare it with simulation.

Case 2: If $A_{1}<A_{2}\left(\lambda_{b}\right)$ then $\lambda_{b} \in\left(0, \frac{\delta \lambda_{u}}{\epsilon B}\right]$ and the optimal transmit power $P^{*}=P_{r}^{*}$. Therefore, the APC at optimal power follows as

$$
\mathcal{P}\left(\lambda_{b}\right)=\frac{\beta \Gamma\left(1+\frac{\alpha}{2}\right)}{b C^{\frac{\alpha}{2}}(\alpha)\left(1-\frac{\delta \lambda_{u}}{B \lambda_{b}}\right)} \frac{1}{\lambda_{b}^{\frac{\alpha}{2}-2}}+P_{o} \lambda_{b} .
$$

We find again the optimum by equating the derivative with respect to $\lambda_{b}$ to zero, i.e.

$$
\begin{aligned}
\frac{d \mathcal{P}\left(\lambda_{b}\right)}{d \lambda_{b}} & =0 \\
\Rightarrow P_{o}-\frac{p \lambda_{b}^{-\frac{\alpha}{2}} \delta^{\prime}}{\left(1-\frac{\delta^{\prime}}{\lambda_{b}}\right)^{2}}+\frac{\left(1-\frac{\alpha}{2}\right) p \lambda_{b}^{2-\alpha / 2}}{1-\frac{\delta^{\prime}}{\lambda_{b}}} & =0,
\end{aligned}
$$

where $p=\frac{\beta \Gamma\left(1+\frac{\alpha}{2}\right)}{b C^{\frac{\alpha}{2}}(\alpha)}$ and $\delta^{\prime}=\delta \frac{\lambda_{u}}{B}$.

Now, (17) can be simplified to the follow equation in $\lambda_{b}$ :

$$
\left.2 P_{\mathrm{o}} \lambda_{b}^{\alpha / 2}\left(\lambda_{b}-\delta\right)^{2}-p(\alpha-4) \lambda_{b}^{3}+p \delta(\alpha-6) \lambda_{b}^{2}\right)=0,
$$

which is a polynomial for $\alpha>4$. The existence of a real solution for the polynomial depends on the value of $\alpha$ and the coefficients.

\section{Simulation Results}

In this section, we numerically plot the results obtained in Sections III and IV and verify them with respect to simulations of our system model. A general remark is that the theoretical results match perfectly the simulated ones. We set up a square of dimension $200 \mathrm{~km} \times 200 \mathrm{~km}$ and the reference user is placed at the center of the square.

In Figs. 2 and 3, we plot the analytical results for the coverage probability and the per-user rate (cf. 5) and compare it with simulations. The two plots demonstrate that both these performance metrics asymptotically saturate to a constant value rather than increasing with BS transmit power increasing. This asserts that increasing transmit power of BSs may not always be the best solution to increase the QoS. This further motivates us to search for the minimum amount of transmit power, which ensures a minimum level of QoS.

In Fig. 4, we compare

- the theoretically derived expression for the approximate optimum power $P_{c}^{*}\left(\lambda_{b}\right)$ given in (9),

- the exact optimum $P_{c}^{*}$, numerically evaluated through exhaustive search for the least value of $P$ that satisfies the coverage constraint of (4), and

- the optimum $P_{c}^{*}$ evaluated using simulations,

as functions of $\lambda_{b}$. It can be noticed that the curves corresponding to theoretical exact minimum and the simulation coincide, whereas expectedly, the approximate theoretical result slightly differs.

Fig. 5 depicts a similar treatment as described in Fig. 4, but for the rate constraint of (4). It can be noticed that the

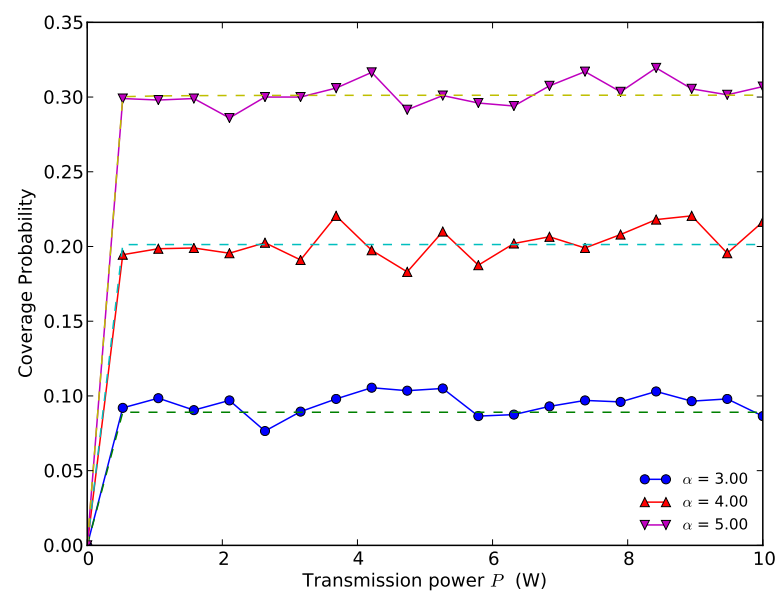

Figure 2. Coverage probability $P_{\text {cov }}$ vs. transmit power $\mathrm{P}(\mathrm{W})$ for different values of $\alpha$ and $\beta=2 \times 10^{-7}$. $P_{\text {cov }}$ asymptotically saturates to a constant value with indefinite increase in $P$.

curves corresponding to theoretical exact minimum and the simulation fairly coincide while the approximate theoretical result is slightly different, as expected. This again validates the correctness of our theoretical analysis.

In Fig. 6, we plot for different values of $\alpha$, the theoretical expression for APC $(\mathcal{P})(14)$ versus BS density $\left(\lambda_{b}\right)$. We compare this with the simulation result where $\mathcal{P}$ is plotted against $\lambda_{b}$ for values of transmit power $(P)$ that satisfy the constraints in 13. Since the bandwidth is reasonably large (of the order of $\left.10^{6} \mathrm{~Hz}\right)$, the region $\lambda_{b} \in\left(0, \frac{\delta \lambda_{u}}{\epsilon B}\right]$ is very narrow and it does not have much of importance. Therefore, we only consider the region $\lambda_{b} \in\left[\frac{\epsilon \lambda_{u}}{\delta B}, \infty\right)$ in our plots. We verify that $\mathcal{P}\left(\lambda_{b}\right)$ has no minima for $\alpha=4$ and it is negligibly small for $\alpha=3$. This is a key message of our work, which dictates a relation between the pathloss exponent and the existence of a minima for the APC $\mathcal{P}$. Furthermore, we observe that in the cases of $\alpha=5$ and 6 deploying too few BSs is not an energy efficient solution.

\section{CONCLUSION}

We have studied the problem of minimizing the power consumption in single-tier cellular wireless networks. Using a low-noise approximation, we derived bounds on the minimum transmit power for achieving certain QoS constraints in terms of coverage and user rate. Based on these optimal transmit power values, we derived the optimal BS density that minimizes the area power consumption subject to minimum coverage probability and per-user rate guarantees. A takeaway message of this paper is that the existence of an optimal BS density for optimizing area power efficiency depends on the specific value of the pathloss exponent.

\section{REFERENCES}

[1] Cisco. (2014) Cisco visual networking index: Global mobile data traffic forecast update, 2013-2018. [Online]. Available: http://goo.gl/177HAJ 


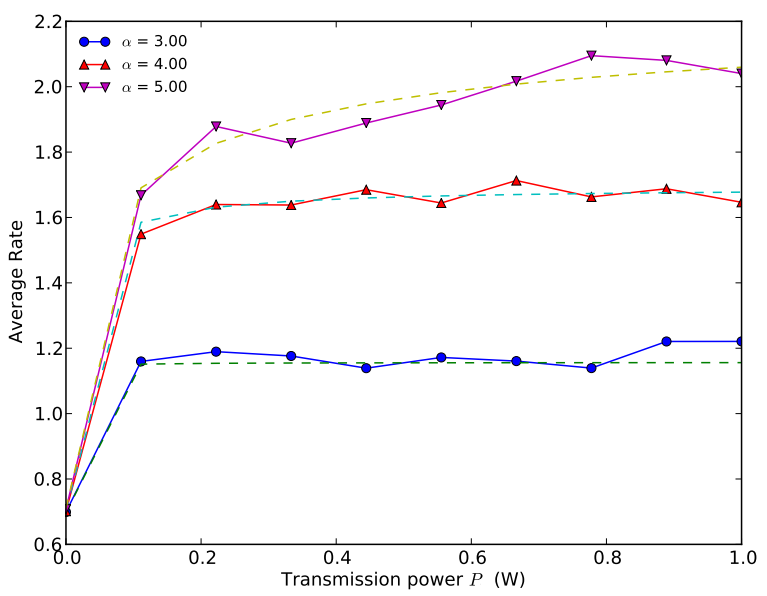

Figure 3. Rate per unit bandwidth $(R)($ bits/Hz) vs. transmit power $P(\mathrm{~W})$ for different values of $\alpha . R$ asymptotically saturates to a constant value with indefinite increase in $P$.

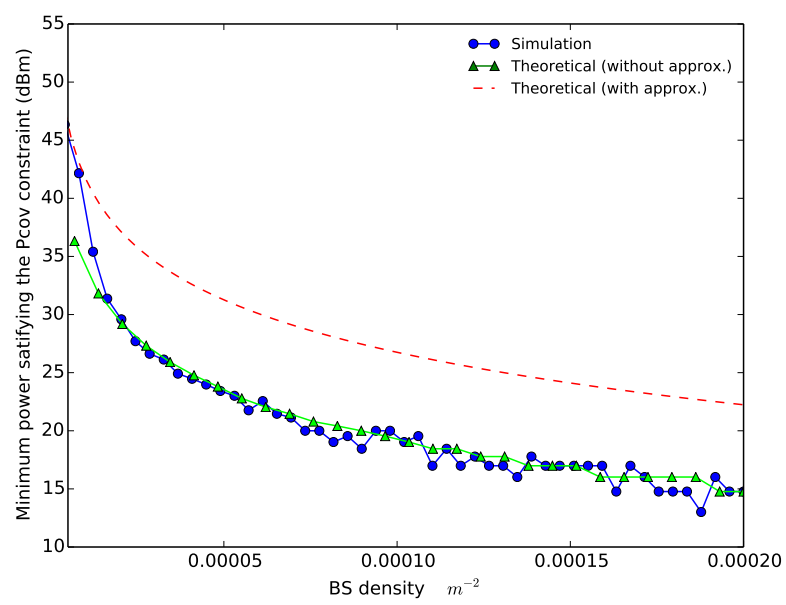

Figure 4. Optimal transmit power satisfying the coverage constraint $\left(P_{c}^{*}\right)$ vs. BS density $\left(\lambda_{b}\right)$ for $\alpha=5, \epsilon=0.6$, and $\beta=2 \times 10^{-7}$.

[2] P. Gonzalez-Brevis, J. Gondzio, Y. Fan, H. Poor, J. Thompson, I. Krikidis, and P.-J. Chung, "Base station location optimization for minimal energy consumption in wireless networks," in Vehicular Technology Conference (VTC Spring), 2011 IEEE 73rd, May 2011, pp. 1-5.

[3] J. G. Andrews, F. Baccelli, and R. K. Ganti, "A tractable approach to coverage and rate in cellular networks," IEEE Transactions on Communications, vol. 59, no. 11, pp. 3122-3134, 2011.

[4] C. S. Chen, V. M. Nguyen, and L. Thomas, "On small cell network deployment: A comparative study of random and grid topologies," in Vehicular Technology Conference (VTC Fall), 2012 IEEE, Sept 2012, pp. 1-5.

[5] S. Mukherjee, Analytical Modeling of Heterogeneous Cellular Networks. Geometry, Coverage, and Capacity. Cambridge, 2014.

[6] F. Richter, A. Fehske, and G. Fettweis, "Energy efficiency aspects of base station deployment strategies for cellular networks," in Proc. IEEE Vehicular Techno. Conf., Anchorage, AL, Sep. 2009, pp. 1-5.

[7] D. Cao, S. Zhou, and Z. Niu, "Optimal base station density for energyefficient heterogeneous cellular networks," in 2012 IEEE International Conference on Communications (ICC), June 2012, pp. 4379-4383.

[8] Y. S. Soh, T. Quek, M. Kountouris, and H. Shin, "Energy efficient heterogeneous cellular networks," IEEE Journal on Selected Areas in

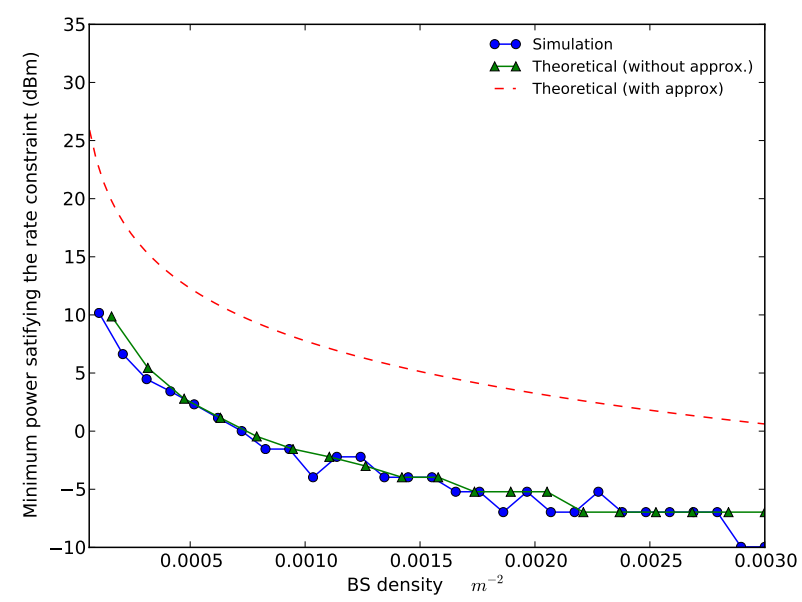

Figure 5. Optimal transmit power satisfying the rate constraint $P_{r}^{*}$ vs. BS density $\lambda_{b}$ for $\alpha=5, \delta=0.6, P_{o}=1 \mathrm{~W}$, and $\beta=2 \times 10^{-7}$.

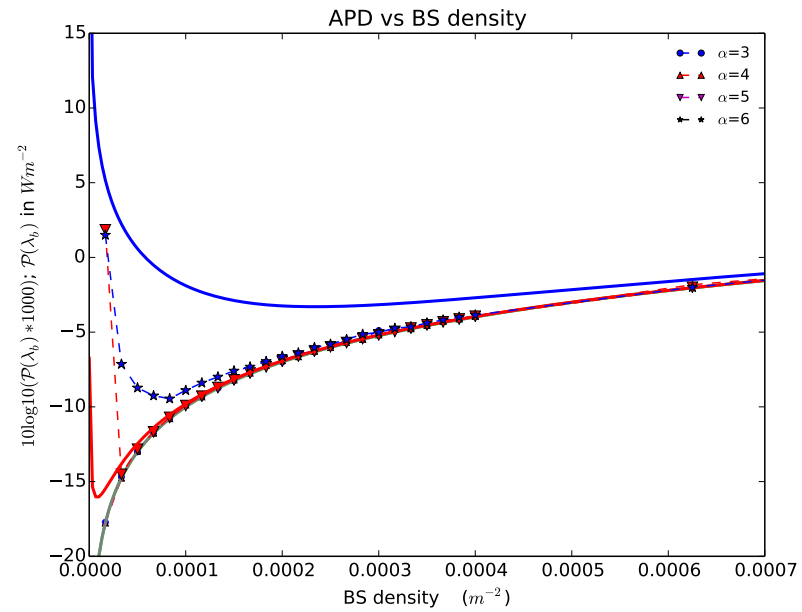

Figure 6. APD vs. $\lambda_{b}$ : Continuous curves represent the theoretical result in (14) and broken curves represent the simulation result of APC for different values of $\alpha . \lambda_{b} \in\left[\frac{\delta \lambda_{u}}{\epsilon B}, \infty\right), \lambda_{u}=1 \mathrm{~m}^{-2}, \gamma=10, \epsilon=0.6, \delta=0.6$ $\beta=2 \times 10^{-7}$, and $B=20 \times 10^{6} \mathrm{M} \mathrm{Hz}$.

Communications, vol. 31, no. 5, pp. 840-850, May 2013.

[9] S. Sarkar, R. K. Ganti, and M. Haenggi, "Optimal base station density for power efficiency in cellular networks," in 2014 IEEE International Conference on Communications (ICC), June 2014.

[10] H. S. Dhillon, R. K. Ganti, F. Baccelli, and J. G. Andrews, "Modeling and analysis of K-tier downlink heterogeneous cellular networks," IEEE Journal on Selected Areas in Communications, vol. 30, no. 3, pp. 550 - 560, Apr. 2012.

[11] T. Samarasinghe, H. Inaltekin, and J. Evans, "Optimal sinr-based coverage in poisson cellular networks with power density constraints," in Vehicular Technology Conference (VTC Fall), 2013 IEEE 78th, Sept 2013, pp. 1-5. 